\title{
Prehypertension and risk for all-cause and cardiovascular mortality by diabetes status: results from the national health and nutrition examination surveys
}

\author{
Yu-Qing Huang ${ }^{1 \#}$, Lin Liu ${ }^{1 \#}$, Jia-Yi Huang ${ }^{1 \#}$, Kenneth Lo ${ }^{1,2}$, Chao-Lei Chen ${ }^{1}$, Yu-Ling Yu ${ }^{1}$, Jie Li $^{1}$, \\ Ying-Qing Feng ${ }^{1}$
}

${ }^{1}$ Department of Cardiology, Guangdong Provincial Key Laboratory of Coronary Heart Disease Prevention, Guangdong Cardiovascular Institute, Guangdong Provincial People's Hospital, Guangdong Academy of Medical Sciences, School of Medicine, South China University of Technology, Guangzhou 510080, China; ${ }^{2}$ Centre for Global Cardiometabolic Health, Department of Epidemiology, Brown University, Providence, USA

Contributions: (I) Conception and design: J Li, YQ Feng; (II) Administrative support: YQ Feng; (III) Provision of study materials or patients: K Lo; (IV) Collection and assembly of data:YQ Huang, L Liu, JY Huang, K Lo, CL Chen, YL Yu; (V) Data analysis and interpretation: YQ Huang, L Liu, JY Huang, K Lo; (VI) Manuscript writing: All authors; (VII) Final approval of manuscript: All authors.

\#These authors contributed equally to this work.

Correspondence to: Ying-Qing Feng; Jie Li. Department of Cardiology, Guangdong Provincial Key Laboratory of Coronary Heart Disease Prevention, Guangdong Cardiovascular Institute, Guangdong Provincial People’s Hospital, Guangdong Academy of Medical Sciences, School of Medicine, South China University of Technology, No. 106, Zhongshan Second Road, Yuexiu District, Guangzhou 510080, China. Email: 651792209@qq.com; leomoku1981@163.com.

Background: It is unclear whether prehypertension alone or combined with diabetes associate with a higher risk for cardiovascular or all-cause mortality. The purpose of this study was to explore the relationship between prehypertension and all-cause and cardiovascular mortality stratified by diabetes status.

Methods: All participants aged $\geq 18$ years were enrolled from the 1999-2014 National Health and Nutrition Examination Surveys (NHANES). Prehypertension was defined as systolic/diastolic blood pressure (BP): 120-139/80-89 mmHg. The status of cardiovascular and all-cause mortality of participants were followed up through 31 December 2015. We performed Cox proportional hazards models and Kaplan-Meier survival curves to evaluate the relationships as mentioned above.

Results: In general, we enrolled 26,070 participants while 15,295 (58.67\%) of them did not have diabetes nor prehypertension, 8,870 (34.02\%) had prehypertension alone, 835 (3.20\%) had diabetes alone, and $1,070(4.11 \%)$ had both diabetes and prehypertension. Compared with participants without diabetes nor prehypertension, the multivariable adjusted hazard ratios and $95 \%$ confidence interval (CI) for allcause mortality among participants with prehypertension alone, diabetes alone, and both diabetes and prehypertension were 1.08 (95\% CI: 0.95, 1.23), $1.26(0.98,1.62)$, and $1.67(1.38,2.03)$ ( $\mathrm{P}$ for trend $<0.001)$, for cardiovascular mortality, the corresponding hazard ratios and 95\% CI were 1.40 (95\% CI: 0.92, 2.14), 2.21 $(1.12,4.38)$, and $2.87(1.65,4.99)$ (P for trend $<0.001)$ respectively.

Conclusions: Our findings suggested that prehypertension associated with an increased risk for cardiovascular and all-cause mortality when compared with diabetes, but not for prehypertension alone. Early screening and intervention for BP among diabetic patients may be beneficial.

Keywords: Prehypertension; diabetes; all-cause mortality; cardiovascular mortality

Submitted Dec 08, 2019. Accepted for publication Feb 04, 2020.

doi: $10.21037 /$ atm.2020.02.144

View this article at: http://dx.doi.org/10.21037/atm.2020.02.144 


\section{Introduction}

Prehypertension, also known as high normal blood pressure (BP), is an intermediate $\mathrm{BP}$ state between normal $\mathrm{BP}$ and hypertension that is characterized by designating systolic $\mathrm{BP}$ (SBP) of 120 to $139 \mathrm{mmHg}$ and diastolic BP of 80 to $89 \mathrm{mmHg}(1,2)$. Prehypertension is a common condition across age, sex, ethnicity, which continues to be a major global health burden (3). A large number of previous studies have shown that prehypertension could significantly increase the risk of hypertension (4), target organ damage (5), cardiovascular events (6), and mortality (7). Meanwhile, data from 1,129,098 participants, which were derived from 20 prospective cohort studies, demonstrated that prehypertension was associated with cardiovascular disease (CVD) mortality, but not with all-cause mortality (8). Another systematic review and meta-analysis of 13 prospective studies with 870,678 participants showed that prehypertension was not associated with all-cause mortality, but was significantly associated with a greater risk of CVD mortality (9). The inconsistency of the above findings indicated the need to investigate whether prehypertension was significantly related to future cardiovascular and allcause mortality. There were several previous studies that have revealed how prehypertension probably increased the risk of CVD events among diabetic patients (10-12). However, a randomized trial performed in United States and Canada, showed that in patients with type 2 diabetes with high risk for cardiovascular events, targeting a SBP of less than $120 \mathrm{mmHg}$, as compared a target with less than $140 \mathrm{mmHg}$, did not reduce the rate of composite major cardiovascular events (13). In addition, there were different views on the therapeutic target of $\mathrm{BP}$ to prevent CVDs or mortality in patients with type 2 diabetes. Therefore, the aim of this study was to investigate the association of prehypertension with cardiovascular and all-cause mortality among United States adults with and without diabetes using data from the National Health and Nutrition Examination Surveys (NHANES) and how the associations were modified by age, gender and ethnic subgroups.

\section{Methods}

\section{Study population}

Data for this study was obtained from the 1999-2014 NHANES, which was an ongoing study designed to assess the health and nutritional status of adults and children in the United States. The NHANES was a nationally representative survey of the civilian, non-institutionalized United States population conducted by the National Center for Health Statistics of the Center for Disease Control and Prevention. Detailed descriptions of the survey design were published elsewhere (14-16). In the 1999-2014 NHANES study, subjects with age $\geq 18$ years old with diagnosis on the status of diabetes and prehypertension were enrolled. However, subjects that aged $<18$ years old, missing data on diabetes history, missing hemoglobin A1c (HbA1C), missing fasting blood glucose (FBG), missing BP, missing mortality data and people with hypertension at baseline were excluded. After applying the exclusion criteria, there were total 26,070 participants included for analysis (Figure 1). The survey protocol was approved by the Institutional Review Board of the Centers for Disease Control and Prevention. All participants have provided written informed consent.

\section{Exposure measures}

Exposure was defined as prehypertension and diabetes. BP measurement was measured by a trained physician using a mercury sphygmomanometer and an appropriately sized cuff. Using diastolic reading as example, if all diastolic readings were zero, the average $\mathrm{BP}$ value would be zero. If there is one diastolic reading was zero with one (or more) non-zero values, the diastolic reading with zero was excluded from BP calculation. If two out of three diastolic readings are zero, the only non-zero diastolic reading was extracted for BP calculation. Two physicians and two health technologists were trained to collect NHANES BP data using a standardized protocol. Hypertension was defined as having a history of hypertension, $\mathrm{SBP} \geq 140 \mathrm{mmHg}$ diastolic BP (DBP) $\geq 90 \mathrm{mmHg}$, and/or using antihypertensive medications (17). Prehypertension was defined as SBP/ DBP: $120-139 / 80-89 \mathrm{mmHg}(1,17)$. Diabetes was defined as FBG $\geq 126 \mathrm{mg} / \mathrm{dL}$ or self-report or taking hypoglycemic agents or $\mathrm{HbA1c} \geq 6.5 \%$ (18).

\section{Covariates}

The questionnaires and test indicators of the NHANES were all obtained based on a standardized procedure and protocol. Age, sex, ethnicity, smoking status, educational level, history of CVD and cancer were self-reported using questionnaire during the in-person interview. Medication history was obtained from self-report and questions for prescribed medications. Smoking status, baseline CVD 


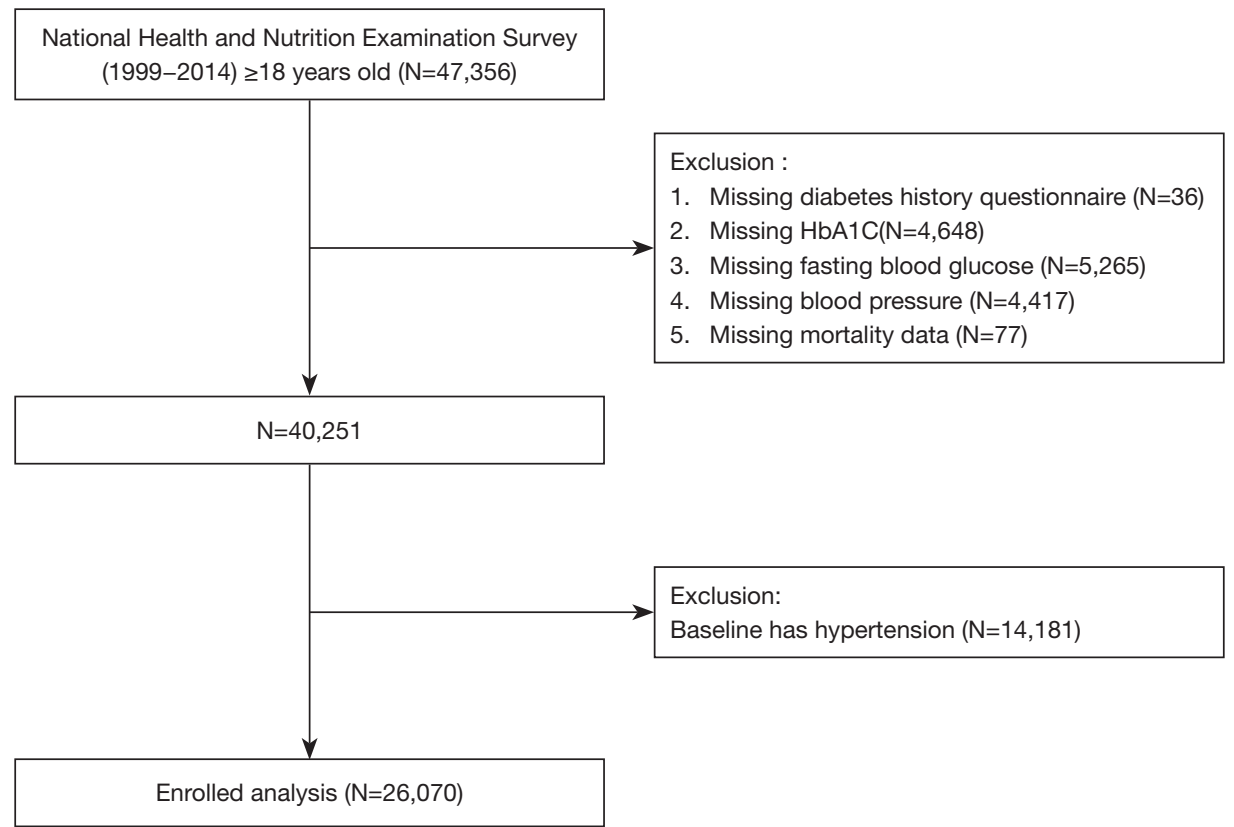

Figure 1 Research flow chart.

and cancer history were dichotomized as yes or no. Educational level was categorized into less than or more than high school education. Other covariates included height, weight, FBG, total cholesterol (TC), high-density lipoprotein cholesterol (HDL-C) and HbA1C were all according to a standardized procedure and protocol. Body mass index (BMI) was defined as mass $(\mathrm{kg})$ divided by the square of height $\left(\mathrm{m}^{2}\right)$. Estimated glomerular filtration rate (eGFR) was calculated using Modification of Diet in Renal Disease formula (19). Detailed examination methods and questionnaire information can be referred to https://wwwn. cdc.gov/nchs/nhanes/Default.aspx.

\section{Outcomes}

The outcomes of the present study were all-cause and cardiovascular mortality. The status of all-cause and cardiovascular mortality was obtained from a publicly available dataset of the NHANES, which captured the vital status and cause of death of survey participants from baseline to 31 December 2015. We examined allcause mortality, as well as mortality due to CVDs. Cardiovascular mortality was defined by International Classification of Diseases, 10th Edition, Clinical Modification System codes (I00-I09, I11, I13, I20-I51) derived from death-certificate data.

\section{Statistical analysis}

Baseline characteristics are presented as mean \pm standard deviation (continuous variables) or percentage (categorical variables) as appropriate. The One-Way ANOVA, Kruskal Wallis $\mathrm{H}$ test and Chi-square tests were used for test for subgroup differences. Participants were grouped into without diabetes and prehypertension, diabetes alone, prehypertension alone and had both diabetes and prehypertension. Survival analysis was performed using standardized Kaplan-Meier curves, and log-rank test was performed to compare the differences in survival rate by prehypertension/diabetic status. Cox proportional hazards regression models were used to calculate hazard ratios (HRs) and 95\% confidence interval (CI) for allcause and cardiovascular mortality. The model 1 only adjusted for prehypertension/diabetic status, and model 2 was additionally adjusted for age and gender. Model 3 was adjusted for age, gender, smoking, education, race, BMI, baseline CVD, baseline status of cancer, TC, HDL-C, eGFR, statin using and antiplatelet drugs. Indicators being used for subgroup analysis included age ( $<70$ or $\geq 70$ years), gender (male and female) and race (White and non-White) and their interactions between diabetes and prehypertension status with all-cause and cardiovascular mortality were also tested. A 2-sided $\mathrm{P}<0.05$ was considered statistically 
Table 1 Baseline characteristics of the participants

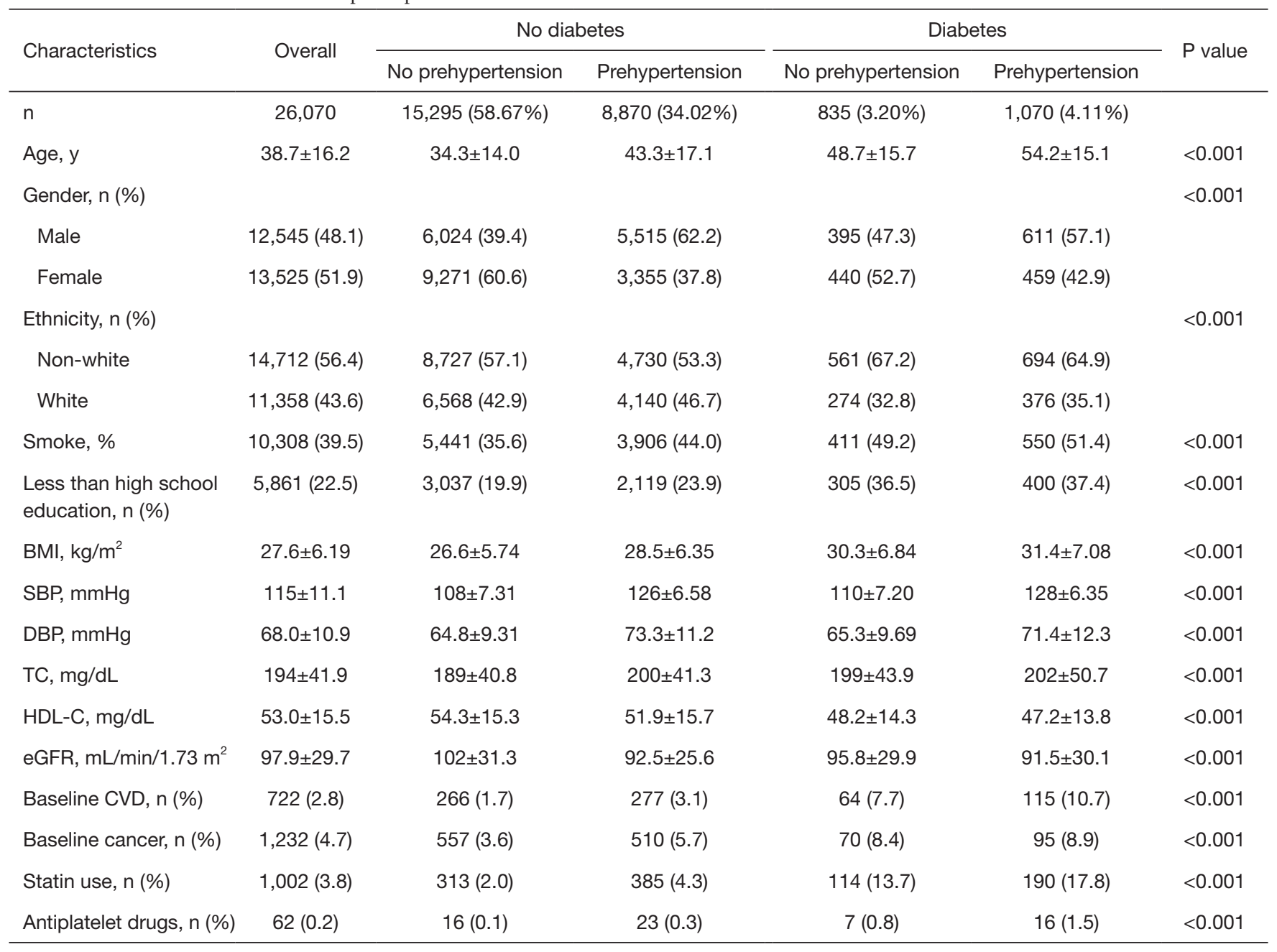

Data are mean \pm SD or percentage. BMI, body mass index; SBP, systolic blood pressure; DBP, diastolic blood pressure; CVD, cardiovascular disease; TC, total cholesterol; HDL-C, high-density lipoprotein cholesterol; eGFR, estimated glomerular filtration rate.

significant. All statistical analyses were performed using $\mathrm{R}$ version 3.3.2 (R Foundation for Statistical Computing, Vienna, Austria).

\section{Results}

\section{Baseline characteristics}

The baseline characteristics of the participants were summarized in Table 1. Among all 26,070 participants, there were 15,295 (58.67\%) participants that did not have diabetes and prehypertension, 8,870 (34.02\%) had prehypertension alone, 835 (3.20\%) had diabetes alone, and 1,070 (4.11\%) had both diabetes and prehypertension, respectively. Participants with both diabetes and prehypertension were found to be older and had higher BMI, had less education and lower levels of eGFR, and were more likely to have smoking history, CVD and cancer at baseline, and taking statin and antiplatelet drugs compared to other groups.

\section{Risk for incident CVD and all-cause mortality}

As shown in Table 2, a total of 1,405 (5.39\%) all-cause mortality and $153(0.59 \%)$ cardiovascular mortality have occurred during the mean follow-up of $8.58 \pm 4.56$ years. There were $506(3.31 \%), 646(7.28 \%), 83(9.94 \%)$ and $170(15.89 \%)$ cases of all-cause mortality, as well as 35 (0.23\%), 78 (0.88\%), 13 (1.56\%) and 27 (2.52\%) cases of CVD mortality occurred in participants without diabetes 
Table 2 All-cause and cardiovascular mortality rate in different groups

\begin{tabular}{|c|c|c|c|c|c|c|c|c|}
\hline Groups & \multicolumn{4}{|c|}{ All-cause mortality } & \multicolumn{4}{|c|}{ Cardiovascular mortality } \\
\hline DM (-) \& preHTN (-) & 506 & 297 (58.7\%) & $209(41.3 \%)$ & $<0.001$ & 35 & 22 (62.9\%) & $13(37.1 \%)$ & 0.008 \\
\hline DM (-) \& preHTN (+) & 646 & $426(65.9 \%)$ & $220(34.1 \%)$ & 0.045 & 78 & $56(71.8 \%)$ & $22(28.2 \%)$ & 0.100 \\
\hline $\mathrm{DM}(+)$ \& preHTN (-) & 83 & $55(66.3 \%)$ & $28(33.7 \%)$ & $<0.001$ & 13 & $11(84.6 \%)$ & $2(15.4 \%)$ & 0.015 \\
\hline Overall & 1,405 & $890(63.3 \%)$ & $515(36.7 \%)$ & $<0.001$ & 153 & $114(74.5 \%)$ & $39(25.5 \%)$ & $<0.001$ \\
\hline
\end{tabular}

Data are percentage. DM, diabetes mellitus; HTN, hypertension.

and prehypertension, had prehypertension alone, had diabetes alone, and had both diabetes and prehypertension groups, respectively. The cumulative survival probability of all-cause and cardiovascular mortality among participants with and without prehypertension as stratified by diabetic status was demonstrated in Figure 2.

As shown in Table 3, when using the group of without diabetes and prehypertension as reference, the Model 1 HRs for all-cause mortality from those with diabetes alone, prehypertension alone, and with both diabetes and prehypertension were $2.20(1.96,2.47), 3.64(2.89,4.6)$ and $5.83(4.9,6.94)$ ( $\mathrm{P}$ for trend was $<0.001)$, and the Model 1 HRs for cardiovascular mortality were 3.84 (2.57, 5.72), $8.29(4.38,15.67)$ and $13.38(8.09,22.11)$ (P for trend was $<0.001)$, respectively. After adjusting for age, gender, smoking, education, race, BMI, baseline CVD, baseline cancer, TC, HDL-C, eGFR, statin using and antiplatelet drugs, the multivariable adjusted HRs for all-cause mortality from those with diabetes alone, prehypertension alone, and with both diabetes and prehypertension were 1.08 $(0.95,1.23), 1.26(0.98,1.62)$ and $1.67(1.38,2.03)$ (P for trend was $<0.001)$, and the HRs for cardiovascular mortality were $1.40(0.92,2.14), 2.21(1.12,4.38)$ and $2.87(1.65,4.99)$ ( $\mathrm{P}$ for trend was $<0.001$ ), respectively.

\section{Subgroups analyses}

As shown in Figure 3, when performing subgroup analysis using age, gender and race, we found that subjects with diabetes and prehypertension have a higher risk for allcause mortality compared to other groups after multivariate adjustment for confounders. However, when analyzing the risk of cardiovascular mortality in different subgroups, we only found that participants with diabetes and hypertension had a higher risk of cardiovascular mortality than other groups (diabetes alone, prehypertension alone, and with both diabetes and prehypertension) among males, people $>70$ years old, White and non-White subgroup. We also found that there was an interaction between race and allcause mortality (P-interaction $=0.003)$, and an interaction between gender and cardiovascular mortality (P-interaction $=0.034)$.

\section{Discussion}

In this study, we found that patients with diabetes and prehypertension had the highest risk of all-cause and cardiovascular mortality compared to those with diabetes alone, prehypertension alone, and without both diabetes and prehypertension. Pre-hypertension was a risk factor for all-cause and cardiovascular death depending on whether the patients had diabetes. Pre-hypertension was an independent risk factor for all-cause and cardiovascular mortality in diabetic patients, but not among non-diabetic patients. In addition, among diabetic patients, there was an age and gender difference in the relationship between prehypertension and risk of cardiovascular mortality. There was an interaction between race and all-cause mortality, and gender has a significant interaction with cardiovascular mortality.

Although our study found that diabetes may elevate the risk of all-cause or cardiovascular death in nonprehypertensive patients compared to those without diabetes and prehypertension, the association was not statistically significant. Our study was inconsistent with a previous study which has demonstrated an approximately four-fold increased risk for cardiovascular events in diabetic individuals with prehypertension compared to those with normal BPs and normal glucose tolerance (11). In addition, we also found that in patients with normal blood glucose, 
$\mathrm{A}$ Groups $+\mathrm{DM}(-) \& \operatorname{preHTN}(-)+\mathrm{DM}(-) \& \operatorname{preHTN}(+)+\mathrm{DM}(+) \& \operatorname{preHTN}(-)+\mathrm{DM}(+) \& \operatorname{preHTN}(+)$

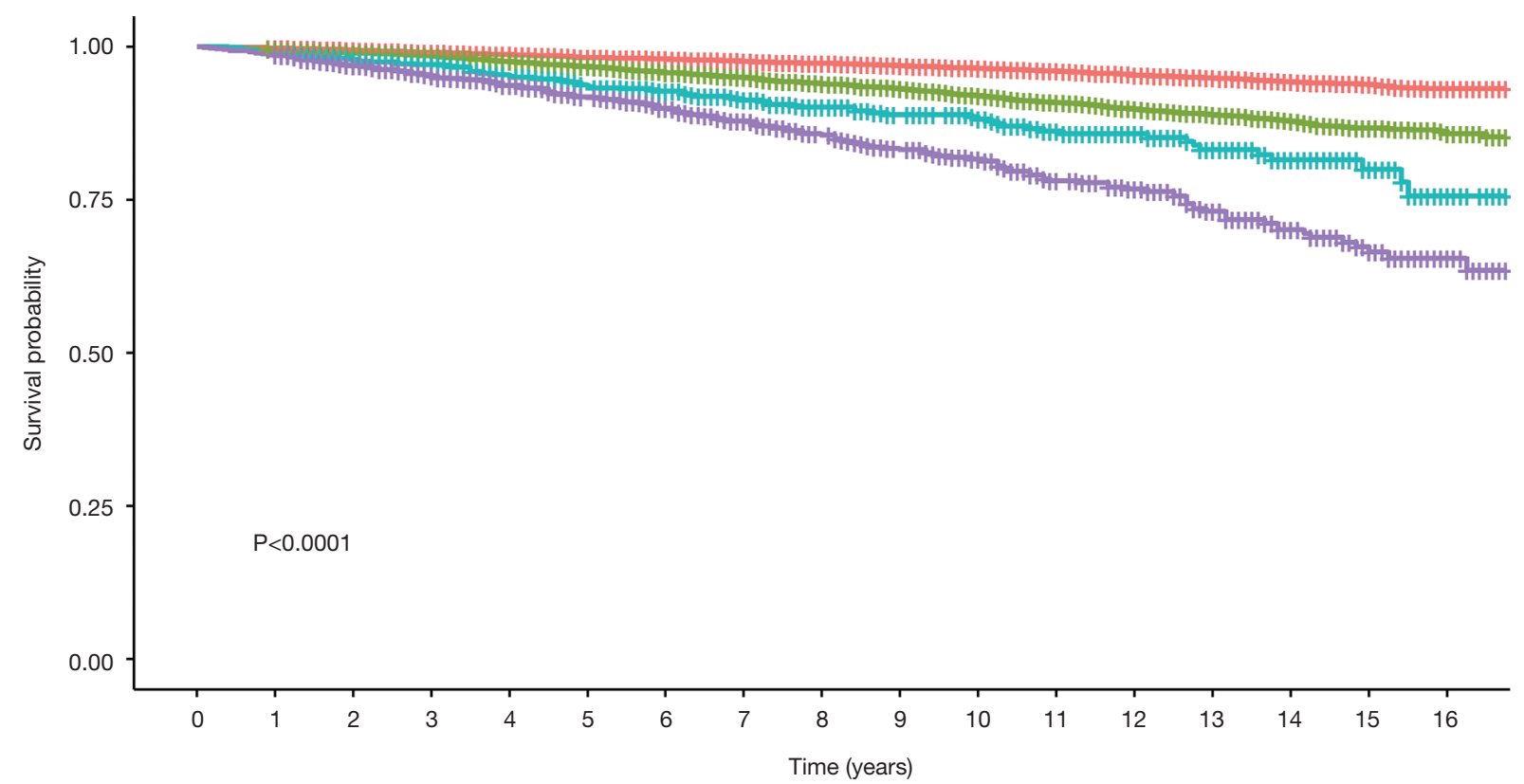

B Groups + DM (-) \& preHTN (-) + DM (-) \& preHTN $(+) \quad+$ DM (+) \& preHTN (-) + DM (+) \& preHTN (+)

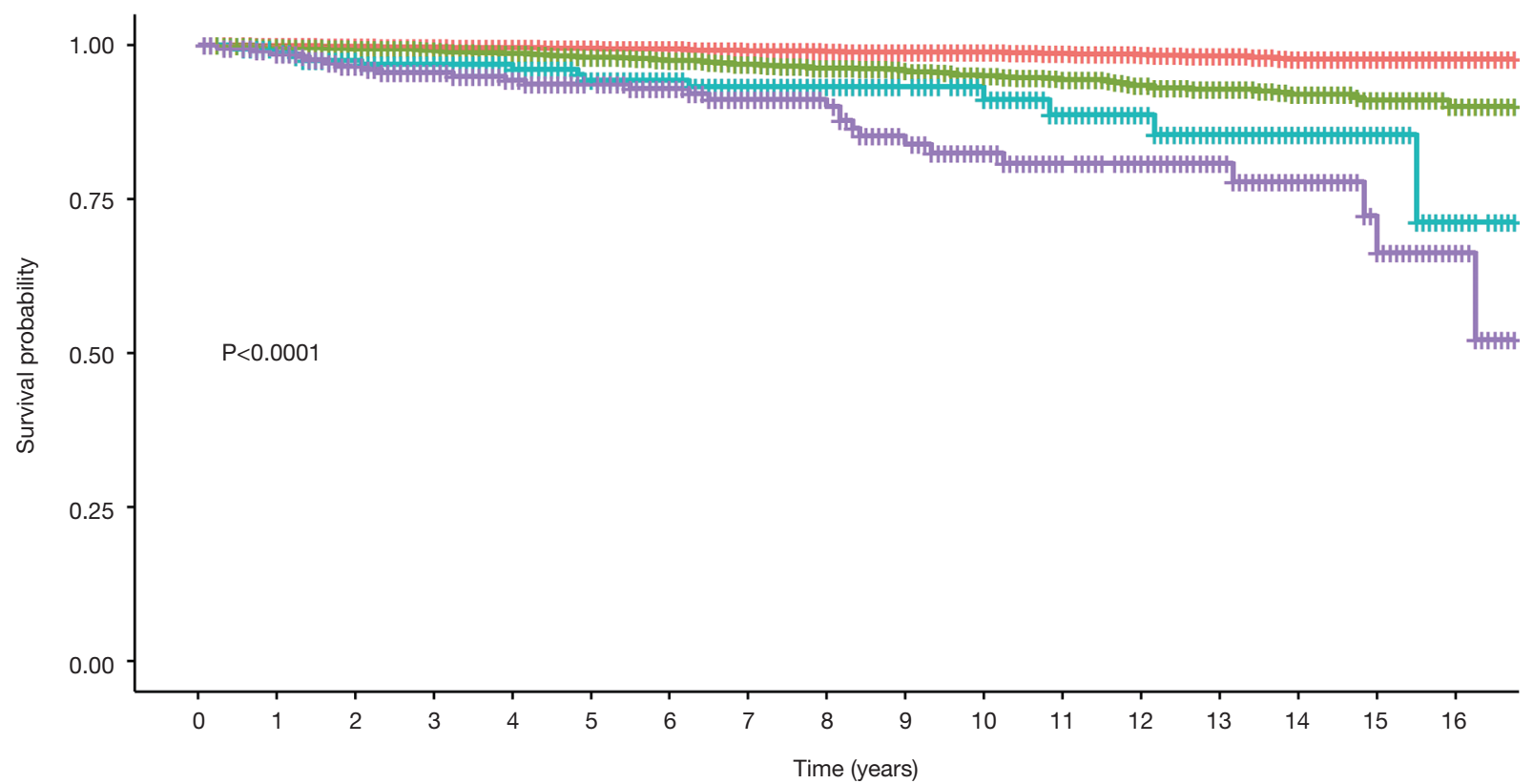

Figure 2 Kaplan-Meier survival curves for all-cause and cardiovascular mortality. DM, diabetes mellitus; preHTN, prehypertension. 
Table 3 Multivariate cox regression analysis for all-cause and cardiovascular mortality in different models

\begin{tabular}{|c|c|c|c|c|}
\hline & Case/total & \multicolumn{3}{|c|}{ HRs (95\% Cl) } \\
\hline \multicolumn{5}{|l|}{ All-cause mortality } \\
\hline DM (-) \& preHTN (-) & $506 / 15,295$ & 1.00 (ref) & 1.00 (ref) & 1.00 (ref) \\
\hline DM (-) \& preHTN (+) & $646 / 8,870$ & $2.20^{\star \star}(1.96,2.47)$ & $1.00(0.88,1.13)$ & $1.08(0.95,1.23)$ \\
\hline DM (+) \& preHTN (+) & $170 / 1,070$ & $5.83^{\star \star}(4.9,6.94)$ & $1.61^{\star \star}(1.34,1.93)$ & $1.67^{\star \star}(1.38,2.03)$ \\
\hline$P$ for trend & & $<0.001$ & $<0.001$ & $<0.001$ \\
\hline \multicolumn{5}{|l|}{ Cardiovascular mortality } \\
\hline DM (-) \& preHTN (-) & $35 / 15,295$ & 1.00 (ref) & 1.00 (ref) & 1.00 (ref) \\
\hline DM (+) \& preHTN (+) & $27 / 1,070$ & $13.38(8.09,22.11)$ & $2.64^{\star \star}(1.58,4.40)$ & $2.87^{\star \star}(1.65,4.99)$ \\
\hline$P$ for trend & & $<0.001$ & $<0.001$ & $<0.001$ \\
\hline
\end{tabular}

Data are HRs and 95\% Cl. DM, diabetes mellitus; HTN, hypertension; HR, hazard ratios; $\mathrm{Cl}$, confidence interval. *, $\mathrm{P}<0.05$; ${ }^{* *}, \mathrm{P}<0.01$. Model 1: unadjusted; Model 2: adjusted for age and gender; Model 3: adjusted for age, gender, smoking, education, race, BMI, baseline CVD, baseline cancer, TC, HDL-C, eGFR, statin using and antiplatelet drugs.

prehypertension could increase the risk of all-cause and cardiovascular death, but the association was also not statistically significant. The finding of our study was not similar to a previous study. Zhang et al. (11) showed that prehypertension might significantly increase the risk of cardiovascular events in people with non-diabetes compared to normotensive population. However, we found that when diabetes was coexisting with prehypertension, it may significantly increase the risk of all-cause and cardiovascular death compared to participants with normal blood sugar and BP. Our research was consistent with the previous studies (20-23). It means that further studies are required to confirm the anticipated benefits of identifying and intervening in persons with prehypertension among diabetic patients.

In addition, in the stratified results by age and gender, we found that participants that had prehypertension alone, and had diabetes alone could increase the risk of all-cause mortality compared to subjects with normal blood glucose with normal BP, but the association was not statistically significant. We also found that racial difference in the relationship between diabetes and all-cause mortality in people with normal BP. Previous studies have also shown that there was a racial difference between diabetes and cardiovascular events or mortality (24-26). Moreover, according to our study, it demonstrated an approximately two-fold increased risk for cardiovascular events in diabetic individuals with prehypertension compared to those with normal BPs and normal glucose tolerance among all the subgroup including age, gender and race. However, there were differences in age, gender, and ethnic groups in the association of diabetes and prehypertension status with cardiovascular mortality. When patients had both diabetes and prehypertension, we showed that males, nonwhite population and those aged $<70$ years had a higher risk of cardiovascular death compared to participants with normal BPs and normal glucose. We speculated that the possible reason was that female hormones probably played a protective role in the occurrence of cardiovascular events among this population. Also, our study found that women have fewer cardiovascular events than men. This suggested that for diabetic patients with prehypertension, whether early intervention in BP may need to be considered separately on different age, gender and ethnicities.

Several limitations should be taken into consideration when interpreting our results. First, FBG and HbA1C were measured only once at baseline, and we did not have data on postprandial blood glucose after 2 hours, which might result in bias in the estimation of the total number of diabetes. Second, we did not adjust for physical activity, which may 
A

\begin{tabular}{|c|c|c|}
\hline \multirow{2}{*}{$\begin{array}{l}\text { Subgroup } \\
\text { Age, y } \\
\text { Age } \geq 70\end{array}$} & & Cases/total \\
\hline & $\begin{array}{l}\text { DM (-) \& preHTN (-) } \\
\text { DM (-) \& preHTN (+) } \\
\text { DM (+) \& preHTN (-) } \\
\text { DM (+) \& preHTN }(+)\end{array}$ & $\begin{array}{l}117 / 357 \\
277 / 735 \\
34 / 82 \\
72 / 181\end{array}$ \\
\hline Age $<70$ & $\begin{array}{l}\text { DM (-) \& preHTN (-) } \\
\text { DM (-) \& preHTN }(+) \\
\text { DM }(+) \text { \& preHTN }(-) \\
\text { DM }(+) \text { \& preHTN }(+)\end{array}$ & $\begin{array}{c}389 / 14938 \\
369 / 8135 \\
49 / 753 \\
98 / 889\end{array}$ \\
\hline male & $\begin{array}{l}\text { DM }(-) \text { \& preHTN }(-) \\
\text { DM }(-) \text { \& preHTN }(+) \\
\text { DM }(+) \text { \& preHTN }(-) \\
\text { DM }(+) \text { \& preHTN }(+)\end{array}$ & $\begin{array}{c}297 / 6024 \\
426 / 5515 \\
55 / 395 \\
112 / 611\end{array}$ \\
\hline Female & $\begin{array}{l}\text { DM (-) \& preHTN (-) } \\
\text { DM (-) \& preHTN }(+) \\
\text { DM (+) \& preHTN }(-) \\
\text { DM }(+) \text { \& preHTN }(+)\end{array}$ & $\begin{array}{c}209 / 9271 \\
220 / 3355 \\
28 / 440 \\
58 / 459\end{array}$ \\
\hline non-white & $\begin{array}{l}\text { DM }(-) \text { \& preHTN }(-) \\
\text { DM }(-) \text { \& preHTN }(+) \\
\text { DM }(+) \text { \& preHTN }(-) \\
\text { DM }(+) \text { \& preHTN }(+)\end{array}$ & $\begin{array}{c}283 / 8727 \\
282 / 4730 \\
48 / 561 \\
92 / 694\end{array}$ \\
\hline White & $\begin{array}{l}\text { DM (-) \& preHTN }(-) \\
\text { DM }(-) \text { \& preHTN }(+) \\
\text { DM }(+) \text { \& preHTN }(+) \\
\text { DM }(+) \text { \& preHTN }(+)\end{array}$ & $\begin{array}{c}223 / 6568 \\
364 / 4140 \\
35 / 274 \\
78 / 376\end{array}$ \\
\hline
\end{tabular}

All-cause mortality

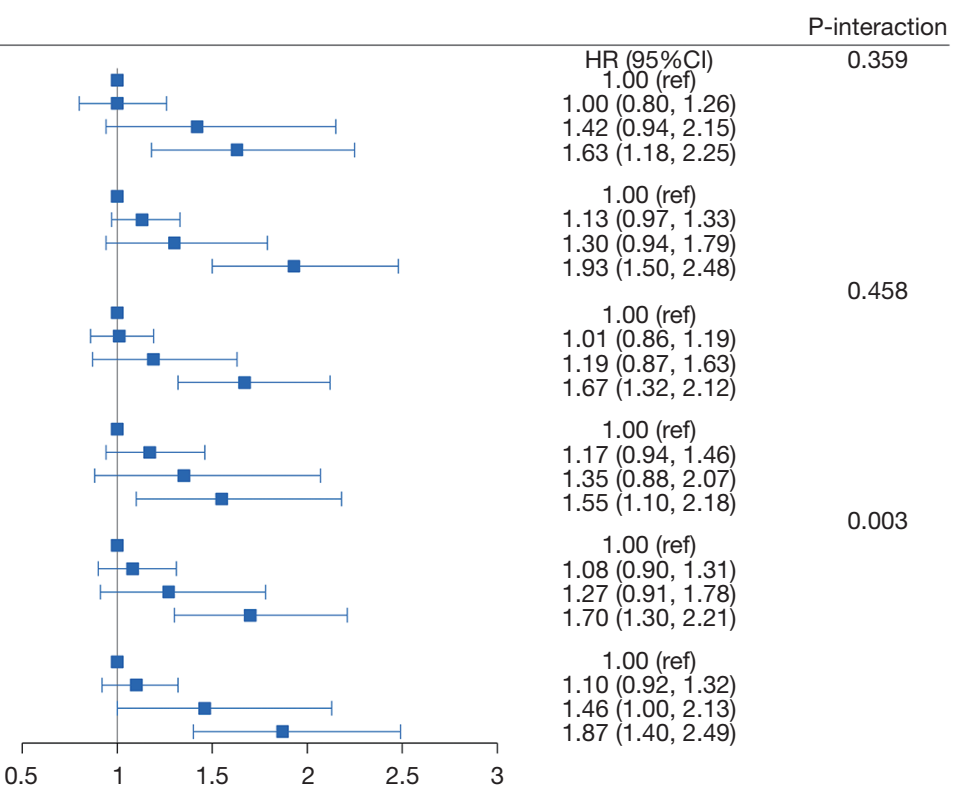

B

Cardiovascular mortality

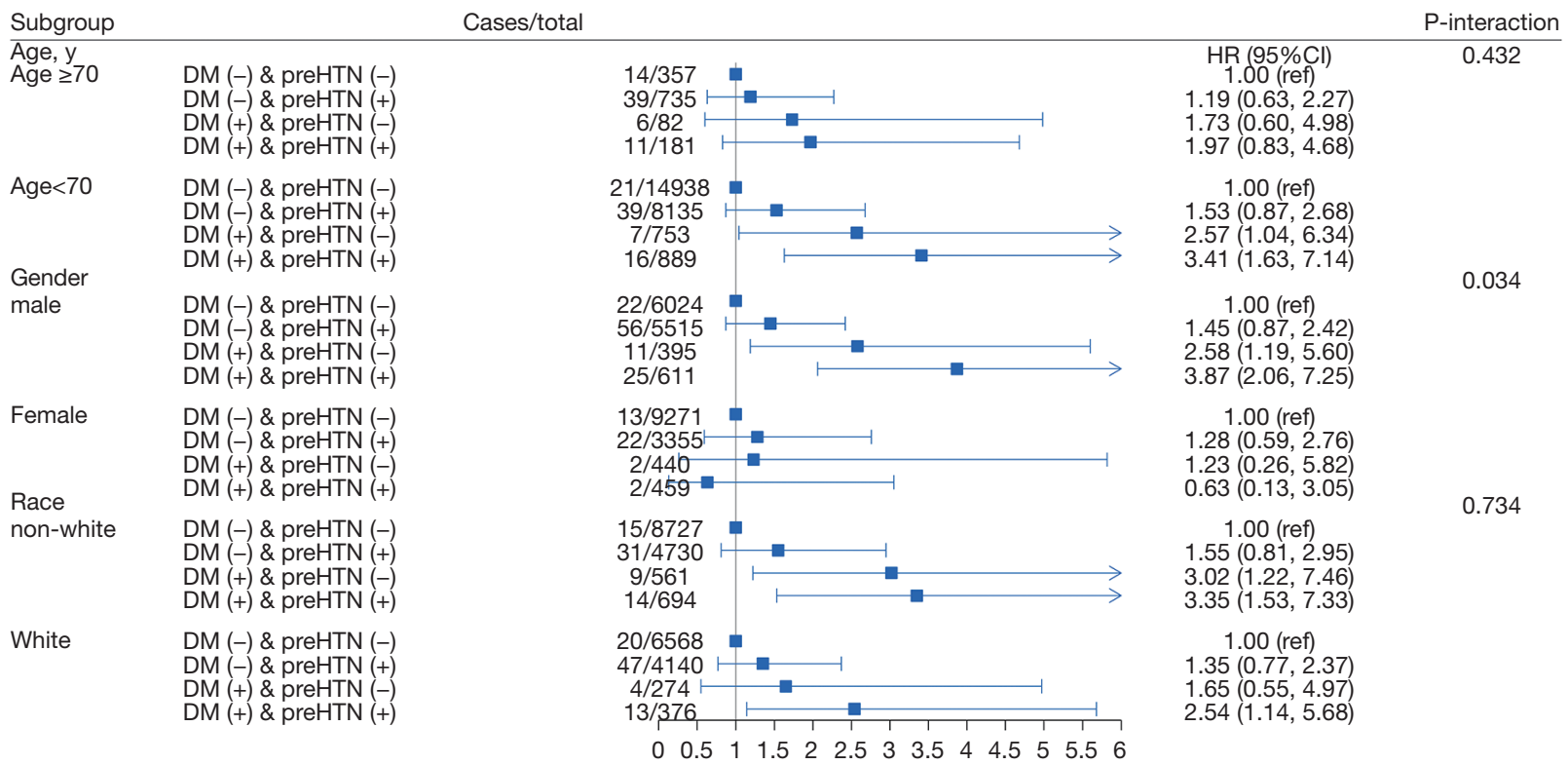

Figure 3 Multivariate cox regression analysis for all-cause and cardiovascular mortality by subgroups. DM, diabetes mellitus; preHTN, prehypertension; HR, hazard ratios; CI, confidence interval.

also associate with cardiovascular and all-cause mortality. In addition, many confounders were self-reported, such as smoking status, previous disease history and medication history, which might be susceptible to self-report bias.
Finally, the types of diabetes were not available within the NHANES data. Despite these limitations, the current study also has several strengths. First, the study obtained data from a nationally representative sample. Second, 
standardized protocols were used for data collection, including BP, HbA1c, and fasting plasma glucose.

In conclusion, prehypertension was significantly associated with an increased risk for cardiovascular and allcause mortality based on diabetic status. In diabetic patients, prehypertension was closely related to cardiovascular and all-cause mortality and was an independent risk factor for them. There were some differences in the association between prehypertension with cardiovascular and all-cause mortality among different subgroups according to age and gender. For diabetic patients, earlier intervention in BP may be beneficial to prevent cardiovascular events and mortality, but some well-designed randomized trials were needed to clarify this situation for diabetic patients in the future.

\section{Acknowledgments}

Funding: This work was supported by the Science and Technology Program of Guangzhou (No. 201803040012), the Science and Technology Program of Guangdong Province (No. 2017B030314041), the National Key Research and Development Program of China (No. 2017YFC1307603, No. 2016YFC1301305) and the Key Area R\&D Program of Guangdong Province (No. 2019B020227005).

\section{Footnote}

Conflicts of Interest: The authors have no conflicts of interest to declare.

Ethical Statement: The authors are accountable for all aspects of the work in ensuring that questions related to the accuracy or integrity of any part of the work are appropriately investigated and resolved. The survey protocol was approved by the Institutional Review Board of the Centers for Disease Control and Prevention (Protocol \#98-12, Protocol \#2005-06, Continuation of Protocol \#2005-06, Protocol \#2011-17, Continuation of Protocol \#2011-17). All participants gave written informed consent.

Open Access Statement: This is an Open Access article distributed in accordance with the Creative Commons Attribution-NonCommercial-NoDerivs 4.0 International License (CC BY-NC-ND 4.0), which permits the noncommercial replication and distribution of the article with the strict proviso that no changes or edits are made and the original work is properly cited (including links to both the formal publication through the relevant DOI and the license). See: https://creativecommons.org/licenses/by-nc-nd/4.0/.

\section{References}

1. Chobanian AV, Bakris GL, Black HR, et al. Seventh report of the Joint National Committee on Prevention, Detection, Evaluation, and Treatment of High Blood Pressure. Hypertension 2003;42:1206-52.

2. Joint Committee for Guideline Revision. 2018 Chinese Guidelines for Prevention and Treatment of Hypertension-A report of the Revision Committee of Chinese Guidelines for Prevention and Treatment of Hypertension. J Geriatr Cardiol 2019;16:182-241.

3. Fernandez C, Sander GE, Giles TD. Prehypertension: Defining the Transitional Phenotype. Curr Hypertens Rep 2016;18:2.

4. Niiranen TJ, Larson MG, McCabe EL, et al. Prognosis of Prehypertension Without Progression to Hypertension. Circulation 2017;136:1262-4.

5. Materson BJ, Garcia-Estrada M, Degraff SB, et al. Prehypertension is real and can be associated with target organ damage. J Am Soc Hypertens 2017;11:704-8.

6. Huang Y, Wang S, Cai X, et al. Prehypertension and incidence of cardiovascular disease: a meta-analysis. Bmc Med 2013;11:177.

7. Wang $\mathrm{S}, \mathrm{Wu} \mathrm{H}$, Zhang Q, et al. Impact of baseline prehypertension on cardiovascular events and all-cause mortality in the general population: a meta-analysis of prospective cohort studies. Int J Cardiol 2013;168:4857-60.

8. Huang Y, Su L, Cai X, et al. Association of all-cause and cardiovascular mortality with prehypertension: a metaanalysis. Am Heart J 2014;167:160-168.e1.

9. Guo X, Zhang X, Zheng L, et al. Prehypertension is not associated with all-cause mortality: a systematic review and meta-analysis of prospective studies. Plos One 2013;8:e61796.

10. Tan JR, Chen YH, Bi YF, et al. Prehypertension is associated with atherosclerosis in Type 2 diabetes. $\mathrm{J}$ Diabetes 2010;2:56-63.

11. Zhang Y, Lee ET, Devereux RB, et al. Prehypertension, diabetes, and cardiovascular disease risk in a populationbased sample: the Strong Heart Study. Hypertension 2006;47:410-4.

12. Kanazawa I, Sugimoto T. Prehypertension increases the risk of atherosclerosis in drug-naive Japanese patients with type 2 diabetes mellitus. PLoS One 2018;13:e0201055.

13. Cushman WC, Evans GW, Byington RP, et al. Effects 
of intensive blood-pressure control in type 2 diabetes mellitus. N Engl J Med 2010;362:1575-85.

14. Fain JA. NHANES. Diabetes Educ 2017;43:151.

15. Kim C, Bullard KM, Herman WH, et al. Association between iron deficiency and A1C Levels among adults without diabetes in the National Health and Nutrition Examination Survey, 1999-2006. Diabetes Care 2010;33:780-5.

16. Palmer MK, Toth PP. Trends in Lipids, Obesity, Metabolic Syndrome, and Diabetes Mellitus in the United States: An NHANES Analysis (2003-2004 to 2013-2014). Obesity (Silver Spring) 2019;27:309-14.

17. Williams B, Mancia G, Spiering W, et al. 2018 ESC/ESH Guidelines for the management of arterial hypertension. Eur Heart J 2018;39:3021-104.

18. The American Diabetes Association. Classification and Diagnosis of Diabetes: Standards of Medical Care in Diabetes-2019. Diabetes Care 2019;42:S13-28.

19. Chancharoenthana W, Wattanatorn S, Vadcharavivad S, et al. Agreement and Precision Analyses of Various Estimated Glomerular Filtration Rate Formulae in Cancer Patients. Sci Rep 2019;9:19356.

20. Oras P, Habel H, Skoglund PH, et al. Elevated Blood Pressure in the Emergency Department: A Risk Factor for Incident Cardiovascular Disease. Hypertension 2020;75:229-36.

Cite this article as: Huang YQ, Liu L, Huang JY, Lo K, Chen CL, Yu YL, Li J, Feng YQ. Prehypertension and risk for all-cause and cardiovascular mortality by diabetes status: results from the national health and nutrition examination surveys. Ann Transl Med 2020;8(6):323. doi:10.21037/atm.2020.02.144
21. Liu N, Yang JJ, Meng R, et al. Associations of blood pressure categories defined by 2017 ACC/AHA guidelines with mortality in China: Pooled results from three prospective cohorts. Eur J Prev Cardiol 2020;27:345-54.

22. Son JS, Choi S, Lee G, et al. Blood Pressure Change from Normal to 2017 ACC/AHA Defined Stage 1 Hypertension and Cardiovascular Risk. J Clin Med 2019. doi: 10.3390/ jcm8060820.

23. Yamagishi K, Sawachi S, Tamakoshi A, et al. Blood pressure levels and risk of cardiovascular disease mortality among Japanese men and women: the Japan Collaborative Cohort Study for Evaluation of Cancer Risk (JACC Study). J Hypertens 2019;37:1366-71.

24. Bellary S, O'Hare JP, Raymond NT, et al. Premature cardiovascular events and mortality in south Asians with type 2 diabetes in the United Kingdom Asian Diabetes Study - effect of ethnicity on risk. Curr Med Res Opin 2010;26:1873-9.

25. Johns E, Sattar N. Cardiovascular and Mortality Risks in Migrant South Asians with Type 2 Diabetes: Are We Winning the Battle? Curr Diab Rep 2017;17:100.

26. Poudel A, Zhou JY, Story D, et al. Diabetes and Associated Cardiovascular Complications in American Indians/ Alaskan Natives: A Review of Risks and Prevention Strategies. J Diabetes Res 2018;2018:2742565. 